\title{
Charged quantum dot micropillar system for deterministic light-matter interactions
}

\author{
P. Androvitsaneas, ${ }^{1}$ A. B. Young, ${ }^{2}$ C. Schneider, ${ }^{3}$ S. Maier, ${ }^{3}$ M. Kamp, ${ }^{3}$ S. Höfling,,${ }^{3,4}$ S. Knauer,,${ }^{1,2}$ \\ E. Harbord, ${ }^{1}$ C. Y. Hu, ${ }^{2}$ J. G. Rarity, ${ }^{2}$ and R. Oulton ${ }^{1,2}$ \\ ${ }^{1}$ Centre for Quantum Photonics, H.H. Wills Physics Laboratory, University of Bristol, Tyndall Avenue, Bristol, BS8 1TL, United Kingdom \\ ${ }^{2}$ Department of Electrical and Electronic Engineering, University of Bristol, Merchant Venturers Building, \\ Woodland Road, Bristol, BS8 1UB, United Kingdom \\ ${ }^{3}$ Technische Physik, Physikalisches Institut and Wilhelm Conrad Röntgen-Center for Complex Material Systems, \\ Universität Würzburg, Am Hubland, 97474 Würzburg, Germany \\ ${ }^{4}$ SUPA, School of Physics and Astronomy, University of St. Andrews, St. Andrews, KY16 9SS, United Kingdom
}

(Received 27 January 2016; revised manuscript received 25 April 2016; published 21 June 2016)

\begin{abstract}
Quantum dots (QDs) are semiconductor nanostructures in which a three-dimensional potential trap produces an electronic quantum confinement, thus mimicking the behavior of single atomic dipole-like transitions. However, unlike atoms, QDs can be incorporated into solid-state photonic devices such as cavities or waveguides that enhance the light-matter interaction. A near unit efficiency light-matter interaction is essential for deterministic, scalable quantum-information (QI) devices. In this limit, a single photon input into the device will undergo a large rotation of the polarization of the light field due to the strong interaction with the QD. In this paper we measure a macroscopic $\left(\sim 6^{\circ}\right)$ phase shift of light as a result of the interaction with a negatively charged QD coupled to a low-quality-factor $(Q \sim 290)$ pillar microcavity. This unexpectedly large rotation angle demonstrates that this simple low- $Q$-factor design would enable near-deterministic light-matter interactions.
\end{abstract}

DOI: 10.1103/PhysRevB.93.241409

The deterministic, lossless exchange of energy between charged QDs and single photons has been shown as the potential building block for a full range of components required for QI and quantum communication [1-3]. A deterministic light-matter interaction would give one the ability to both switch the photon state with a high fidelity as well as keep photon loss near zero (high efficiency). To achieve these simultaneously, it is essential that all the photon energy that couples to and from the quantum emitter must do so almost exclusively within a well-defined electromagnetic mode, where one can input/collect single photons. Input/output coupling efficiency is parametrized by the $\beta$ factor, the ratio between the rate of coupling of the dipole to this well-defined mode compared to the total coupling rate of the dipole to all available electromagnetic modes, including leaky ones.

Great success has been had in approaching this limit in several systems, including photonic crystal (PC) waveguides [4] and photonic nanowires [5]. For optical cavities, however, this limit has proven difficult to approach. Light-matter interaction strengths in the "strong coupling" regime have been achieved for high- $Q$-factor pillar microcavities [6] and in photonic crystal cavities [7], and could show high fidelity switching. However, the input/output mode is usually not well defined in high- $Q$-factor cavities: the escape rate to and from a well-defined input channel is similar to the escape rate to leaky cavity modes (CMs). These leaky modes arise either from the intrinsic design of the structure or from fabrication imperfections, putting a limit on the efficiency of high- $Q$ factor microcavities where the escape rate into the input/output mode is slow by design. However, in a low- $Q$-factor pillar the cavity lifetime is very short. Thus one may easily design a high- $\beta$-factor structure with a well-defined input/output mode, a crucial advantage [8].

The $\beta$ factor is directly linked to the competition between the rates of coherent and incoherent interactions present in these structures. The coherent coupling rate $\Gamma$ is related to the parameter $g$, which represents the dipole cavity field coupling rate in the Jaynes-Cummings Hamiltonian [9]. In the case where the dipole is resonant with the cavity, this leads to a modified rate of emission given by $\Gamma=4 g^{2} / \kappa$, where $\kappa$ represents the decay rate of the $\mathrm{CM}$. The incoherent fraction is parametrized by $\gamma$ and $\gamma^{*}$, where $\gamma$ represents the rate at which the dipole radiatively couples to other available non-CMs [see Fig. 1(a)], and $\gamma^{*}$ represents the pure dephasing rate of the dipole. The $\beta$ factor is now defined as $\beta=\frac{\Gamma}{\Gamma+\gamma+\gamma^{*}}$, the ratio of the rate of coherent interaction to the total interaction rate. In order to achieve high $\beta$ factors, one may either increase $g$ by decreasing the mode volume of the cavity (as $g \propto 1 / \sqrt{V_{\text {eff }}}$ ), or $\kappa$ which is inversely proportional to the $Q$ factor of the cavity. However, one can also modify $\gamma$ geometrically by reducing the number of available vacuum modes into which the dipole can decay [10]. This has been exploited in PCs to redistribute the rates of emission, enabling the design [11] and realization [4] of high- $\beta$-factor PC waveguides. Here we show that this same redistribution occurs in low $Q$-factor micropillars. This contrasts with the conventional approach for micropillar cavities where the $\beta$ factor is increased via the strong enhancement of the decay rate into the CM (Purcell enhancement) [8].

A direct measure of the interaction strength is the magnitude of the phase shift induced on photons as they coherently scatter from the dipole transition [12]. It is a well-known quantum optics phenomenon [13] that in the limit where $\beta \sim 1$ the light will experience a $\pi$ phase shift relative to the incident light $[14,15]$, the maximum possible in this configuration. In fact, it has been shown that as long as $\beta>0.5$ then the maximum possible phase shift of $\pi$ will always be observed [16]. Thus a high-fidelity operation (i.e., a $\pi$ phase shift) will always be observed for $\beta>0.5$, while increasing the $\beta$ factor further increases the efficiency. Thus high efficiency and fidelity may be achieved when $\beta \sim 1$. 
(a)

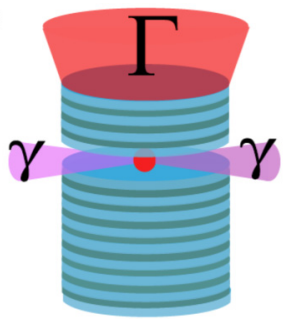

(c)

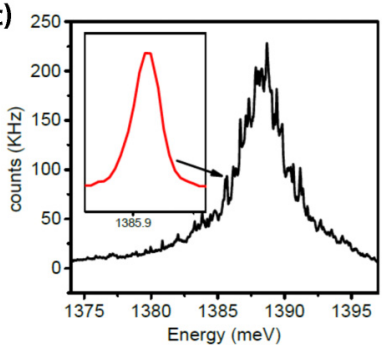

(b)

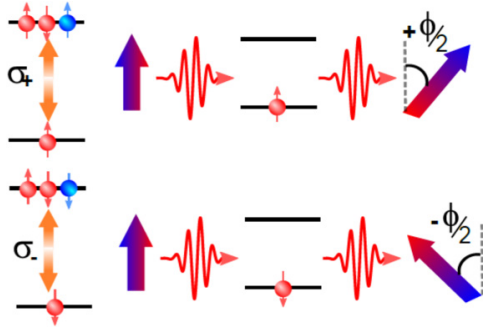

(d)

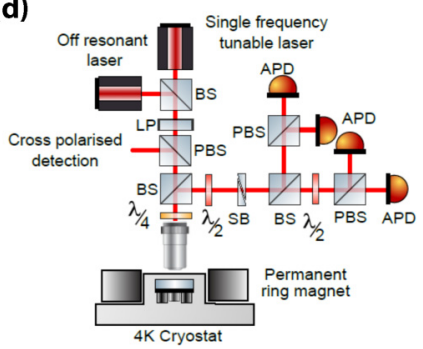

FIG. 1. (a) Schematic of the QD micropillar system with the available decay channels. (b) Available circular transitions for a negatively charged QD and the corresponding excitons created, along with the corresponding rotation of linearly polarized coherently scattered photons. (c) Photoluminescence (PL) spectrum of the micropillar under consideration at $T=12 \mathrm{~K}$. The $\mathrm{CM}$ may be seen and the QD on top of the half maximum point of the CM. Inset: PL spectrum of the QD under lower off-resonant excitation power. (d) Schematic of the experiment. The laser polarization is set with a linear polarizer (LP). The measured light is split on a nonpolarizing beamsplitter (BS) into two different measurement paths. The first path, for the identification of the transitions, is selected using a polarizing beamsplitter (PBS) for the cross-polarized resonant scattering (detection path on the left). The second path is incident on a phase shift interferometer setup (on the right), where a quarter wave plate $(\lambda / 4$, QWP) and a half wave plate $(\lambda / 2$, HWP) are used to rotate the light to the correct measurement basis and a Soleil-Babinet compensator ( $\mathrm{SB}$ ) is used to account for any birefringence present and to calibrate our interferometer. Avalanche photodiodes (APDs) are used to record the signal.

In this work we consider a negatively charged QD containing a dopant electron. This has spin-selective transitions. If the excess electron is in the spin-up (-down) state, only $\sigma^{+}$right $\left(\sigma^{-}\right.$left $)$circular polarized light can scatter from the dipole [see Fig. 1(b)]. Accordingly, if the light is in a superposition of an interacting part and a noninteracting part, the induced phase shift will be picked up by the interacting component, while the noninteracting part has no phase shift. For example, if vertically $|V\rangle$ polarized light scatters off a charged QD, the interacting part will acquire a phase shift $\phi$ dependent on the strength of the interaction, and the orientation of the QD spin [i.e., $|V\rangle|\downarrow\rangle \rightarrow\left(e^{i \phi}\left|\sigma^{-}\right\rangle-\left|\sigma^{+}\right\rangle\right)|\downarrow\rangle$ and $\left.|V\rangle|\uparrow\rangle \rightarrow\left(\left|\sigma^{-}\right\rangle-e^{i \phi}\left|\sigma^{+}\right\rangle\right)|\uparrow\rangle\right]$ [17-19]. This phase shift now maps onto a rotation along the linear polarization plane. This is known as the spin-dependant Kerr (Faraday) rotation $\phi_{r}$, with $\phi_{r}=\phi / 2$. Thus, for $\beta>0.5$ the maximum $\phi_{r}=\pi / 2$ will be achieved, i.e., a rotation from $|V\rangle$ to $|H\rangle$ [16].

We study a QD incorporated into the center of a low- $Q$ factor $2-\mu \mathrm{m}$-diameter micropillar cavity consisting of a $\lambda$-thick cavity surrounded by two distributed Bragg reflectors (DBRs)

and with a CM $Q$ factor of $\sim 290$. The DBR structure consists of 18 (5) bottom (top) AlAs/GaAs mirror pairs. The $\operatorname{In}(\mathrm{Ga}) \mathrm{As}$ QD layer is modulation doped. The QD transition we examine in this work can be seen in Fig. 1(c), where it is spectrally detuned by $\sim 2.7 \mathrm{meV}$ with respect to the $\mathrm{CM}$ resonance (slightly below the half maximum of the $\mathrm{CM}$ ). In order to define two circularly polarized transitions we introduce a magnetic field (500 mT) along the growth direction (Faraday geometry). The resonant scattering (RS) from the QD was measured using dark field microscopy techniques [20], where we tune a linearly polarized single-frequency laser (1-s integration at each frequency) over the QD from the blue to the red side and collect the orthogonal linear polarization at a power of $\sim 1 \%$ of the saturation power. The QD has been identified as charged performing RS under different polarization bases. The crosspolarized RS signal of the Zeeman split doublet is shown in Fig. 2(a), with an observed scattering response over $\sim 4.5 \mu \mathrm{eV}$. In order to measure the phase we perform polarization analysis of the total RS signal, detecting simultaneously horizontal $(|H\rangle)$, vertical $(|V\rangle)$, diagonal $(|D\rangle)$, and antidiagonal $(|A\rangle)$ [see Fig. 1(d)]. The phase shift $\phi$ can then be obtained using

$$
\sin \phi=\frac{D-A}{2 \sqrt{H V}} .
$$

The measured phase shift response is shown in Fig. 2(b), where a maximum phase shift of $\phi \sim 6^{\circ}$ is observed, corresponding to a Kerr rotation of $\phi_{r} \sim 3^{\circ}$. The measured Kerr rotation angle is similar in magnitude to that reported by Arnold et al. [21]. The similarity in phase-shift values is surprising since the $Q$-factor value of $\sim 2000$ used in Ref. [21] is an order of magnitude larger. Naively, the only difference between the high- and low- $Q$-factor micropillar would be the factor of 10 increase in photon loss rate $(\kappa)$, resulting in a reduction of $\Gamma$ by an order of magnitude. Therefore, the measured phase shift from the low- $Q$ micropillar would be of the order of mrad [22], assuming approximately the same value of $g$ in both cases. The macroscopic phase shift we have demonstrated indicates that this description of the system is inadequate.

To better understand this result we need to examine the sources of decoherence in our system. Figure 3(a) shows the QD lifetime measured under pulsed $p$-shell excitation showing a lifetime of $0.82 \pm 0.02 \mathrm{~ns}$, corresponding to a Fourier transform-limited linewidth of $\Gamma_{t}=(\Gamma+\gamma) \sim 0.8 \mu \mathrm{eV}$. Clearly, this is significantly narrower than the $4.5 \mu \mathrm{eV}$ measured using RS in Fig. 2(a). The measured RS linewidth corresponds to the convolution of the Fourier limited spectral response, pure dephasing, and any "spectral jitter" present. Previous studies of QD RS have shown this broadening to be almost exclusively a product of spectral wander (jitter) as a result of charge and spin noise. By measuring the RS linewidth at a rate of 10's of $\mathrm{kHz}$, almost transform-limited RS linewidths have been observed [23]. This suggests that the contribution from pure dephasing, occurring on time scales shorter than the QD radiative lifetime, is not significant. We confirm this in our case by measuring the first- and second-order correlation function $\left[\mathrm{g}^{(1)}(t), \mathrm{g}^{(2)}(t)\right.$; see Supplemental Material [24]], of the cross-polarized RS photons. We increase the input laser intensity and measure a decay in the $\mathrm{g}^{(1)}(t)$ corresponding to a coherence time of $\sim 5 \mathrm{~ns}$, indicating $\gamma^{*} \ll \Gamma, \gamma$. Furthermore, 

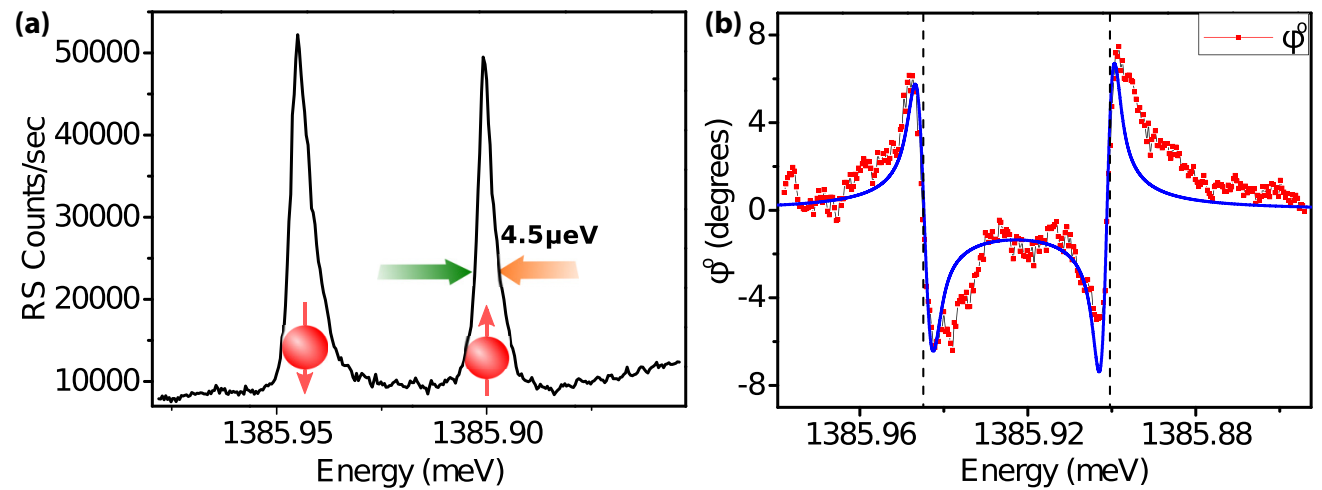

FIG. 2. (a) Photon counts using the resonant scattering technique detected in the cross-polarized arm. The two circular transitions are indicated, with arrows to indicate the spin orientation. (b) Experimental data for the phase shift (red points) overlaid with the theoretical fit (blue line) after incorporating jitter and accounting for the thermalization between the spin states. The dashed lines correspond to the resonant frequency for each transition.

measurement of the autocorrelation $g^{(2)}(t)$ reveals bunching of the signal on time scales of order $\mu$ s, indicative of the spectral wander, in agreement with Kuhlmann et al. [23].

The effect of spectral wander will be to significantly wash out the observed phase features in Fig. 2(b). A stochastic model may be used to describe this. We assume the QD transition has a $\Gamma_{t}=0.8 \mu \mathrm{eV}$, with the resonance moving about a central frequency following a Gaussian profile, which agrees with the RS line shape in Fig. 2(a). By applying this model to the phase-shift data in Fig. 2(b), using the value of $\Gamma_{t}$ obtained from Fig. 3(a), we can fit for one free parameter: $\Gamma$ (see Supplemental Material [24]). The result is the fit (blue line) in Fig. 2(b) which gives $\Gamma \approx 0.52 \mu \mathrm{eV}$, resulting in a high $\beta$ factor of $\beta \approx 0.65 \pm 0.03$. The only additional assumption here is that the spin is in a thermal state, and in the time averaged measurement we perform (1-s integration time) each spin state is occupied only $50 \%$ of the time. This effectively limits the maximum observable phase shift to $\phi=\pi / 2$ for this measurement. This value for $\Gamma$ implies that the radiative
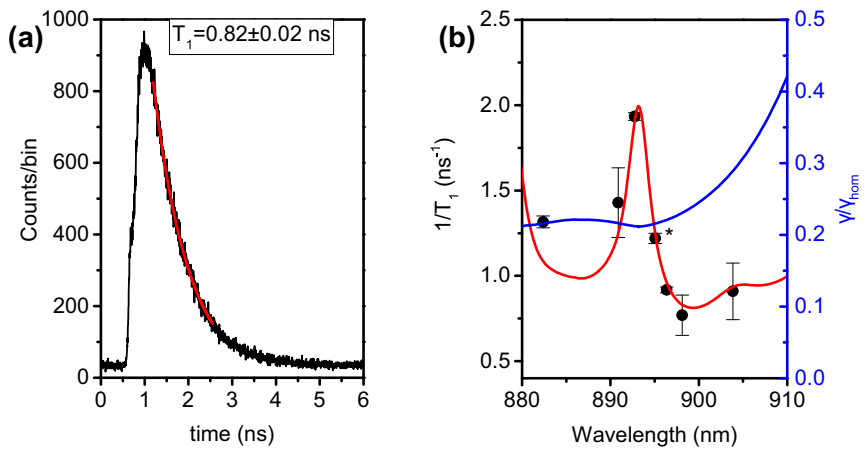

FIG. 3. (a) Measured lifetime of the QD transition under study. (b) The black circles represent the $1 / T_{1}$ quantity. The black star next to the black circle in the middle denotes the QD under study. The lifetimes where measured under pulsed $p$-shell excitation. $\Gamma_{t} / \gamma_{\text {hom }}$ was simulated and the simulated value for $1 / T_{1}$ is plotted fitting for $T_{1 \mathrm{hom}}=710 \mathrm{ps}$ (red curve). $T_{1 \mathrm{hom}}=710 \mathrm{ps}$ is in agreement with lifetime measurements performed on similar wavelength QDs without an etched structure from the same wafer. The blue curve is the ratio $\gamma / \gamma_{\text {hom }}$ as it is simulated for this structure. decay rate into lossy modes is only $\gamma \approx 0.28 \mu \mathrm{eV}$. This is much smaller than the decay rate in homogeneous material (or free space) $\gamma_{\text {hom }}(\sim 1 \mu \mathrm{eV})$, typically used as a value for $\gamma$ in these equations.

This is perhaps not surprising when one considers the geometry of the micropillar cavity. In conventional atomcavity QED where the cavities are macroscopic, the CM only subtends a small angle, leaving almost $4 \pi$ steradians of possible decay modes contributing to $\gamma$. This geometrical limitation leads to the assumption that $\gamma$ is similar to the free space decay rate $\gamma_{\text {hom }}$ [13]. Under this assumption, the only approach to achieving a high $\beta$ factor is an enhancement of the decay rate into the CM (Purcell enhancement) compared to homogeneous material [8,25-27]. However, for the wavelength-scale micropillar cavity used here, the geometry is very different from an atom cavity. A simple geometric approximation allows one to estimate that only $0.12 \times 4 \pi$ steradians can escape below the critical angle from the side of the pillar (i.e., $\gamma=0.12 \gamma \mathrm{hom}$ ). This is clearly an oversimplification, but regardless, it elucidates the underlying physics; in the real system our fits give a value $\gamma \sim 0.3 \gamma$ hom [where $\gamma_{\text {hom }}=0.93 \mu \mathrm{eV}$ based on Fig. 3(b)]. A commercialgrade simulator based on the finite-difference time-domain (FDTD) method was used to perform the calculations [28]. The simulated side losses [FDTD, blue curve in Fig. 3(b)] predict $\gamma \sim 0.22 \gamma \mathrm{hom}$. The discrepancy may be attributed to incoherent contributions from higher-order modes or coupling through the bottom mirrors, but still the dominant contribution is from the side losses.

The fact that this micropillar shows a high $\beta$ factor for QDs close to resonance agrees well with previous calculations [29] and is confirmed in our simulations. Furthermore our simulations show that for a QD resonant with the CM, only $\sim 15 \%$ of the total emission from the QD radiatively couples out of the side of the micropillar $\left(\gamma=0.15 \Gamma_{t}\right)$. The QD in question here is, however, slightly detuned from the cavity mode, $\sim 0.66$ cavity linewidths. Naturally, the $\beta$ factor is frequency dependent: $\beta(\omega)=\frac{\Gamma(\omega)}{\Gamma(\omega)+\gamma(\omega)}$, where $\Gamma(\omega)=\frac{4 g^{2}}{\kappa\left\{1+\left[2\left(\omega_{c}-\omega\right) / \kappa\right]^{2}\right\}}$, with $\omega_{c}$ and $\omega$ the cavity and emitter resonances, respectively [13]. Hence one obtains a higher $\beta$ factor for an on-resonant QD $\left(\omega=\omega_{c}\right)$ : we predict for this micropillar $\beta\left(\omega_{c}\right)=0.85$ [see Fig. 3(b)], compared to the 
studied QD with $\beta \approx 0.65$. Note that the criterion for $\beta$ factor $>0.5$ holds even for a detuned QD, obtaining the maximum phase shift of $\pi$.

The frequency dependence of $\beta$ might imply, as the QD is strongly detuned, that the QD lifetime should increase dramatically. This has never been observed experimentally, as $\gamma$ is not independent of $\omega$. Figure 3(b) shows that the calculated side leakage $\gamma / \gamma_{\text {hom }}$ actually increases as the QD is detuned from the cavity. We deduce that by strongly detuning a QD from the cavity mode, the DBRs now act as a stop band to create efficient waveguiding out of the side of the micropillars, hence increasing $\gamma(\omega)$ relative to $\gamma\left(\omega_{c}\right)$, evidenced by the blue line in Fig. 3(b) (see also Supplemental Material).

This work demonstrates an ideal design for a micropillar; however, photonic design does not alleviate the problems with the properties of the emitter itself, such as phonon dephasing and spectral jitter. Nevertheless, we have shown that pure dephasing of the transition due to phonons is minimal. Spectral wander is also not insurmountable. The time scales for the spectral jitter as a result of charge noise are around a 1-100 $\mu \mathrm{s}$ [23]. This is slow compared to typical spin coherence times of the order $\mu \mathrm{s}$ [30]. Thus, single-shot or time-resolved Kerr rotation measurements [22] as opposed to the time-averaged experiment shown here, would allow observation of the maximum $\phi=\pi$ phase shift achievable with this particular QD-cavity combination. This should enable us to generate spin-photon entanglement within the spin coherence time with a $50 \%$ probability, compared to $0.003 \%$ in the current state of the art [31]. Hence efficient spin-photon entanglement using such a low- $Q$-factor design is indeed feasible.

In summary we show that a low- $Q$-factor micropillar cavity meets the requirements for unity fidelity $(\beta$ factor of $>0.5$ ), in an intrinsically high-efficiency system. Strong light-matter interaction is inferred from a measurement of the input photon phase shift of $\sim 6^{\circ}$. Pure dephasing in this system is minimal, but the magnitude of the phase shift is nevertheless reduced due to easily quantifiable spectral jitter. By fitting to this spectral jitter and taking into account the thermal state of the spin and QD-cavity detuning we estimate the measured $\beta$ factor to be $\sim 0.65$, with potential to allow a full $\pi$ phase shift of incident light if spectral jitter could be overcome. Previous QD-microcavity designs usually compromise efficiency for fidelity: for this simple-to-fabricate low- $Q$-factor micropillar design, fidelity and efficiency go hand-in-hand, thus enabling useful spin-photon entanglement devices.

The authors thank H. F. Hofmann for helpful discussions. This work was funded by the Future Emerging Technologies (FET) programme within the Seventh Framework Programme for Research of the European Commission, FET-Open, FP7284743 [project Spin Photon Angular Momentum Transfer for Quantum Enabled Technologies (SPANGL4Q)] and the German Ministry of Education and research (BMBF) and Engineering and Physical Sciences Research Council (EPSRC) [project Solid State Quantum Networks (SSQN)]. J.G.R. is sponsored by the EPSRC fellowship EP/M024458/1.
[1] L.-M. Duan and H. J. Kimble, Phys. Rev. Lett. 92, 127902 (2004).

[2] C. Y. Hu, A. Young, J. L. O’Brien, W. J. Munro, and J. G. Rarity, Phys. Rev. B 78, 085307 (2008).

[3] A. M. Stephens, Z. W. E. Evans, S. J. Devitt, A. D. Greentree, A. G. Fowler, W. J. Munro, J. L. O'Brien, K. Nemoto, and L. C. L. Hollenberg, Phys. Rev. A 78, 032318 (2008).

[4] T. Lund-Hansen, S. Stobbe, B. Julsgaard, H. Thyrrestrup, T. Sünner, M. Kamp, A. Forchel, and P. Lodahl, Phys. Rev. Lett. 101, 113903 (2008)

[5] J. Claudon, J. Bleuse, N. S. Malik, M. Bazin, P. Jaffrennou, N. Gregersen, C. Sauvan, P. Lalanne, and J.-M. Gérard, Nat. Photon. 4, 174 (2010).

[6] J. P. Reithmaier et al., Nature 432, 197 (2004).

[7] T. Yoshie, A. Scherer, J. Hendrickson, G. Khitrova, H. M. Gibbs, G. Rupper, C. Ell, O. B. Shchekin, and D. G. Deppe, Nature 432, 200 (2004).

[8] W. L. Barnes, G. Björk, J. M. Gérard, P. Jonsson, J. A. E. Wasey, P. T. Worthing, and V. Zwiller, Eur. Phys. J. D 18, 197 (2002).

[9] E. T. Jaynes and F. W. Cummings, Proc. IEEE 51, 89 (1963).

[10] S. Noda, M. Fujita, and T. Asano, Nat. Photon 1, 449 (2007).

[11] G. Lecamp, P. Lalanne, and J. P. Hugonin, Phys. Rev. Lett. 99, 023902 (2007).

[12] A. B. Young, R. Oulton, C. Y. Hu, A. C. T. Thijssen, C. Schneider, S. Reitzenstein, M. Kamp, S. Höfling, L. Worschech, A. Forchel et al., Phys. Rev. A 84, 011803 (2011).
[13] H. J. Carmichael, Statistical Methods in Quantum Optics 2 (Springer-Verlag, Berlin, Heidelberg, 2008).

[14] P. Kochan and H. J. Carmichael, Phys. Rev. A 50, 1700 (1994).

[15] G. Zumofen, N. M. Mojarad, V. Sandoghdar, and M. Agio, Phys. Rev. Lett. 101, 180404 (2008).

[16] H. F. Hofmann, K. Kojima, S. Takeuchi, and K. Sasaki, J. Opt. B 5, 218 (2003).

[17] M. Atature, J. Dreiser, A. Badolato, and A. Imamoglu, Nat. Phys. 3, 101 (2007).

[18] J. Berezovsky, M. H. Mikkelsen, O. Gywat, N. G. Stoltz, L. A. Coldren, and D. D. Awschalom, Science 314, 1916 (2006).

[19] A. Reiserer, S. Ritter, and G. Rempe, Science 342, 1349 (2013).

[20] A. V. Kuhlmann, J. Houel, D. Brunner, A. Ludwig, D. Reuter, A. D. Wieck, and R. J. Warburton, Rev. Sci. Instrum. 84, 073905 (2013).

[21] C. Arnold, J. Demory, V. Loo, A. Lemaître, I. Sagnes, M. Glazov, O. Krebs, P. Voisin, P. Senellart, and L. Lanco, Nat. Commun. 6, 6236 (2015).

[22] Y. Q. Li, D. W. Steuerman, J. Berezovsky, D. S. Seferos, G. C. Bazan, and D. D. Awschalom, Appl. Phys. Lett. 88, 193126 (2006).

[23] A. V. Kuhlmann, J. H. Prechtel, J. Houel, A. Ludwig, D. Reuter, A. D. Wieck, and R. J. Warburton, Nat. Commun. 6, 8204 (2015).

[24] See Supplemental Material at http://link.aps.org/supplemental/ 10.1103/PhysRevB.93.241409 for details on the cavity QED modeling and spectral wander modeling are provided. Also further sample information, characterization measurements, and simulations on the properties of the photonic structure are given. 
[25] A. Musiał, C. Hopfmann, T. Heindel, C. Gies, M. Florian, H. A. M. Leymann, A. Foerster, C. Schneider, F. Jahnke, S. Höfling et al., Phys. Rev. B 91, 205310 (2015).

[26] J. M. Gérard, B. Sermage, B. Gayral, B. Legrand, E. Costard, and V. Thierry-Mieg, Phys. Rev. Lett. 81, 1110 (1998).

[27] A. Auffeves-Garnier, C. Simon, J.-M. Gerard, and J.-P. Poizat, Phys. Rev. A 75, 053823 (2007).

[28] Lumerical Solutions, Inc (http://www.lumerical.com/tcadproducts/fdtd/).
[29] Y. L. D. Ho, T. Cao, P. S. Ivanov, M. J. Cryan, I. J. Craddock, C. J. Railton, and J. G. Rarity, IEEE J. Quantum Electron. 43, 462 (2007).

[30] A. Greilich, D. R. Yakovlev, A. Shabaev, A. L. Efros, I. A. Yugova, R. Oulton, V. Stavarache, D. Reuter, A. Wieck, and M. Bayer, Science 313, 341 (2006).

[31] J. R. Schaibley, A. P. Burgers, G. A. McCracken, L. M. Duan, P. R. Berman, D. G. Steel, A. S. Bracker, D. Gammon, and L. J. Sham, Phys. Rev. Lett. 110, 167401 (2013). 